\title{
Description of Radar Target Using Resonance Based Null Polarization
}

\author{
Faisal Aldhubaib \\ Electronics Department, College of Technological Studies, Public Authority of Applied Education, Kuwait
}

\begin{abstract}
By the representation of the target transient response as a series of natural resonance modes, a target feature set based on the co-polarized null states of the modes is used to represent a radar target. This is done by incorporating the mode residue terms in a scattering matrix of quadrature polarization channels, and then bythe optimization (using the Lagrangian optimization procedure) of the received power (determined by the Kennaugh and Stokes formulism), the characteristic polarization state set of a mode is obtained. A $2 D$ plane model is used to demonstrate the ability of this concept to convey a complex target physical attributes such as elongation, symmetry and tilt; the results showed that a feature set based on nulls states of the target modes is robust.
\end{abstract}

Keywords: Kennaugh Matrix, Resonance Theory, Polarization, Co-pol null, Target feature set

\section{Introduction}

When identifying radar targets, researchers have used resonance based feature set [1-4], and sometime incorporated along a polarimetric feature to enhance performance [5-12]. As the target works as a polarization transformer, features such as symmetry, elongation and tilt that are related to the target shape can be deduced from the target characteristic polarization states (CPS). The CPS set represents the polarization states of the sensing antenna at which maximum, minimum, or null power conditions at the antenna serminal exist. For a co-polarized (co-pol) reception, there will be four CPS, namely two co-pol maximums and two co-pol nulls. These four states will form a polarization fork shape around a great circle on a polarization sphere, i.e. Poincare sphere [13, 14]. Of which, the null states should be able to unfold the elongation, symmetry and the tilt degrees of the target along the direction of the sensing antenna.

However, the concept of using CPS to characterize a radar target is well developed in the narrowband context. This narrowband limitation can be tied to the fact that the target polarization response is a function of frequency and therefore spectrally non-uniform depolarization exists if impulsive illumination and reception are used. Note, this is unlike the spectral spread due to noise, clutter, or even refraction in the transmission medium; all of which can be handled by ensemble or time averaging achieved by means of pulse integration $[15,16]$. To extend the CPS concept into a broadband context, a sparse representation (signature based-model) approach is required to find terms from the target transient response that are time-invariant, and at the same time can relate robustly to the target composite and shape. To achieve this, the singularity theory, as defined in the Singularity Expansion Method (SEM)[17], is exploited. According to SEM, the late time portion of the temporal response from a perfect electric conducting (PEC) body can be represented in the time domain as a series of resonance modes with the target natural frequencies and associated residues. The resonant frequency terms are related to the target dimensions and have enhanced returns, and also independent of aspect and polarization directions[18]. On the other hand, the residue terms are dependent on aspect and polarization directions, and thus carry the polarization characteristic of the target. Henceforth, the CPS can be derived from the complex residues, and subsequently, information about the target composite and shape can be inferred from the co-null set. So the contribution of this paper is to validate the feasibility of using the co-pol nulls of resonance modes to describe radar targets.

\section{Formulation}

\subsection{Polarization Representation: SEM Context}

If a radar target is illuminated by a sufficiently broadband radio wave, with bandwidth covering the target dominant resonance modes, a suitable extraction algorithm, such as the Method Pencil-of-Function (MPOF), should be capable of recovering the target resonance modes from the late time portion of the backscattered temporalresponse. According to the SEM model, the late time of the target impulse response can be expressed as a sum of damped complex exponentials, with dominant terms relating to the dimensions and composition of the target.

For a quadrature polarization directions, the backscattered response set $\mathrm{a}_{\mathrm{t}}$ in any orthogonal linear basis, e.g. (x,y), forms $\mathrm{a} \mathrm{R}^{2 \times 2}$ matrix, and according to SEM model will be expressed as follows

$$
\mathbf{a}_{t}=\sum_{n=1}^{M} \mathbf{C}_{n} e^{-\left(\sigma_{n}+j \omega_{n}\right) t}
$$

Such that

$$
\mathbf{C}_{n}=\left[\begin{array}{cc}
c_{n}^{x x} & c_{n}^{x y} \\
c_{n}^{y x} & c_{n}^{y y}
\end{array}\right]
$$

Here $t>T_{\mathrm{L}}$, and $T_{\mathrm{L}}$ denotes the late time onset after which the incident wave has totally passed the target. The mode terms $\sigma$, wand $c$ denote the: damping factor, resonant frequency and complex residue. The modal order $M$ gives the number of modes presumably excited. The subscripts denote the transmitter and receiver polarization directions, where $x x$ and $y y$ denote the co-polarized scattering directions or channels, 


\section{International Journal of Science and Research (IJSR) \\ ISSN (Online): 2319-7064}

Index Copernicus Value (2013): 6.14 | Impact Factor (2015): 6.391

while $x y$ and $y x$ denote the cross-polarized scattering channels (reciprocal for monostatic case). Since the polarization information is embedded in the mode residues, the time dependence term can be omitted.

\subsection{Stokes vector and Kennaugh Matrix}

The Stokes vector is used to describe the polarization state of a wave or antenna for completely (and even partially) polarized waves as follows

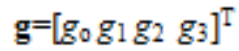

Subject to: $g_{\mathrm{o}}=\left(g_{1}{ }^{2}+g_{2}{ }^{2}+g_{3}{ }^{2}\right)^{1 / 2}$ for fully polarized wave. ${ }^{\mathrm{T}}$ denotes the transpose. The $\mathrm{g}_{\mathrm{o}}$ denotes the wave intensity or total instantaneous power, $\mathrm{g}_{1}$ gives the portion of the wave that is horizontally or vertically polarized, $\mathrm{g}_{2}$ gives the portion of the wave that is linearly oriented at $\pm 45^{\circ}$ and $g_{3}$ gives the portion of the wave that is left or right circularly polarized, respectively [16]. In the case of a partially polarized wave due to clutter or noise, ensemble-time averaging of Stokes vector is used.

In general, the second moment properties (i.e. power terms) of a residue matrix $\mathbf{C}$ at a single mode can be represented by the Kennaugh formulism, as follows [17]

$$
[\mathbf{K}]=\mathbf{R}\left(\left|[\mathbf{C}] \otimes[\mathbf{C}]^{*}\right|\right) \mathbf{R}^{-1}, \quad \mathbf{R}^{-1}=\frac{1}{2} \mathbf{R}^{* T}
$$

Here

$$
\mathbf{R}=\left(\begin{array}{cccc}
1 & 0 & 0 & 1 \\
1 & 0 & 0 & -1 \\
0 & 1 & 1 & 0 \\
0 & j & -j & 0
\end{array}\right)
$$

The product $\left(\left|[\mathbf{C}] \otimes[\mathbf{C}]^{*}\right|\right)$ gives the entire correlation relations between the co- and $\mathrm{x}$-pol residues of a mode, and is defined as the Kronecker product of the $\mathbf{C}$ matrix and its conjugate. The Kennaugh matrix $\mathbf{K}$ relates incident and scattered Stokes vectors $\mathbf{g}_{\mathbf{i}}$ and $\mathbf{g}_{\mathrm{s}}$ as follows

$$
g_{s}=K \cdot g_{i}
$$

\subsection{Received Power}

For backscattering and in the reciprocal case, the use of one antenna for transmitting and receiving is adequate to give maximum power and no greater power can be received in separate antennas for transmitting and receiving. Thus the one antenna case (monostatic case) is assumed, where the antenna Stokes vector $\mathbf{g}_{\mathbf{a}}$ is identical for transmit and receive and consequently the incident wave Stokes vector $\mathbf{g}_{\mathbf{i}}$ is identical to the antenna Stokes vector $\mathbf{g}_{\mathrm{a}}$. Therefore, the received power at the receiver terminals can be expressed in terms of $\mathbf{g}_{\mathbf{i}}$ and $\mathbf{K}$ as

$$
P=\mathrm{g}_{\mathrm{i}} \cdot[\mathrm{K}] \cdot \mathrm{g}_{\mathrm{i}}^{\mathrm{T}}
$$

The associated received power in the co-pol channel is

$$
P_{\mathrm{c}}=\mathrm{g}_{\mathrm{a}} \cdot\left[\mathrm{K}_{\mathrm{c}}\right] \cdot \mathrm{g}_{\mathrm{a}}{ }^{\mathrm{T}}
$$

$$
\left[\mathbf{K}_{\mathrm{c}}\right]=\frac{1}{2}\left(\begin{array}{cccc}
1 & 0 & 0 & 0 \\
0 & 1 & 0 & 0 \\
0 & 0 & 1 & 0 \\
0 & 0 & 0 & -1
\end{array}\right) .[\mathrm{K}]
$$

Here the subscript , $\mathrm{C}^{\text {ce }}$ denotes the co-pol configuration. Equation (7) is essential for deriving the antenna co-pol CPS and their associated power levels using the Lagrange multiplier method.

\subsection{Characteristic Polarization States}

For the one antenna case (monostatic case), optimization involves properly choosing the antenna polarization states such that power developed at the receiving antenna terminals is maximum, minimum, or null for a given resonance mode. The power optimization will be carried out for the co-pol only, since all the target physical attributes can be inferred from the co-pol CPS. Without loss of generalization, the received power in (7) can be maximized and minimized by applying the Lagrangian multiplier method to the antenna Stokes vector $\mathbf{g}_{\mathrm{a}}$ with the constraint that the transmit power is normalized to unity. The constraint condition $\Phi$ written in terms of the Stokes sub-variables is then defined as

$$
\Phi\left(g_{1}, g_{2}, g_{3}\right)=\sqrt{g_{1}^{2}+g_{2}^{2}+g_{3}^{2}}-1=0
$$

The variations of the antenna Stokes variables $\left(\mathrm{g}_{1}, \mathrm{~g}_{2}, \mathrm{~g}_{3}\right)$ will lead to maximizing or minimizing the received power at the reception terminals, where the optimum co-pol powers can be found by simultaneously solving for the first partial derivatives of (9) subject to the constraint condition in (8).This procedure results in three simultaneous partial derivative equations as follows:

$$
\frac{\partial P_{c}}{\partial g_{m}}-\mu \frac{\partial \Phi}{\partial g_{m}}=0, \quad m=1,2,3
$$

Here $\mu$ is the Lagrangian multiplier and gives the rate of change of the power quantity being optimized as a function of the constraint variables. Solving (9) produces two pairs of co-pol CPS. These are the orthogonal (antipodal) co-pol max pair $\left(\mathrm{cm}_{1}, \mathrm{~cm}_{2}\right)$ and the co-pol null pair $\left(\mathrm{cn}_{1}, \mathrm{cn}_{2}\right)$.

\subsection{Elongation, Symmetry and Tilt Degrees}

First, the elongation (vs. roundness) degree is determined by taking the dot product of the null vectors $\left(\left|g_{\mathrm{cn} 1} \bullet \mathrm{g}_{\mathrm{cn} 2}\right|\right)$. A value of one represent a totally long and thin shaped target, e.g. wire, and a value of zero represent a totally round shaped target, e.g. sphere. Second, by taking the element-by-element sum of the two co-null Stokes vectors, i.e. $\left[\mathrm{g}\left(\mathrm{cn}_{1}\right) .+\mathrm{g}\left(\mathrm{cn}_{2}\right)\right]$, the symmetry degree is determined by taking the $1 / 2 \arcsin \left(g_{3}\right)$ of the sum vector, such that $0^{\circ}$ represent a symmetrical target and $\pm 45^{\circ}$ represent a totally asymmetrical target. Finally, the tilt degree is determined by taking the $1 / 2 \arctan \left(g_{2} / g_{1}\right)$ of the sum vector

Where 


\section{International Journal of Science and Research (IJSR) \\ ISSN (Online): 2319-7064}

Index Copernicus Value (2013): 6.14 | Impact Factor (2015): 6.391

\section{Simulation and Results}

To validate the concept of describing the target by the co-null state set in a resonance mode context, a numerical example using a 2D wire model of aircraft is implemented. The modeled aircraft and the simulation values are depicted in Figureland Table I, respectively. The simulated backscattered frequency-domain data were generated by method of moments algorithm (MoM) using FEKO [19]. The resultant backscattered was filtered by a Gaussian window to create the effect of a Gaussian shaped impulse and then the frequency data were transformed to the time-domain by Fourier transform.

Table I: Simulation values

\begin{tabular}{|c|c|}
\hline parameter & Value \\
\hline start Frequency & $1.9 \mathrm{MHz}$ \\
\hline stop Frequency & $1 \mathrm{GHz}$ \\
\hline number of frequency Points & 512 \\
\hline excitation source voltage & $1 \mathrm{~V}$ \\
\hline incidence direction & along u3-axis (normal) \\
\hline wire radius & $0.33 \mathrm{~cm}$ \\
\hline number of segments & 83 \\
\hline SEM modal order & 4 \\
\hline late-time on-set & $10 \mathrm{~ns}$ \\
\hline
\end{tabular}

Applying the MPOF to the FFT time response, four resonances are found approximately at the following frequencies: $150.4, \quad 294.9, \quad 441.5$ and 519.5 MHz.Figure2shows that using the MoM, these resonances are found to correspond to mainly tosections of the mid, the nose-wings, the wings and the tails, respectively. The corresponding complex residues are depicted inTable II. Inserting the complex residues into (4) for each mode and then inserting each respective $\mathrm{K}_{\mathrm{c}}$ into (7) and applying(9) and then solving, the co-pol null state set $\mathrm{cn}_{1}$ and $\mathrm{cn}_{2}$ at each mode is derived and listed in Table III. The co-pol null set at each resonanceindicates that the target has different null set, and subsequently, different physical attributes. At the first resoanance, the geometry is forcasted to be highly long (as $\left.\left|\mathrm{g}_{\mathrm{cn} 1} \bullet \mathrm{g}_{\mathrm{cn} 2}\right|=1\right)$ and tilted about $45^{\circ}\left(\right.$ as $\left.1 / 2 \cdot \arctan \left(\mathrm{g}_{2} / \mathrm{g}_{1}\right)=45^{\circ}\right)$; and thus corresponding mainly to the mid section. As for the second to the fourth, they have similar co-null sets with dihedral property as $\left|\mathrm{g}_{\mathrm{cn} 1} \bullet \mathrm{g}_{\mathrm{cn} 2}\right| \neq 1$, forecasting a wing or a tail structure. However, all modes indicate that the target composite is symmetrical and $45^{\circ}$ tilted. These physical attributes of the co-null at the dominant modes totally agree with what is a priori known about the targetes shape.

Table II:The Scattering coefficients in terms of the Complex residues.

\begin{tabular}{|c|c|c|c|}
\hline Mode order & $c_{11}$ & $\mathrm{c}_{12}$ & $c_{22}$ \\
\hline $1^{\text {st }}$ & $0.49-.13 \mathrm{j}$ & $0.37-0.38 \mathrm{j}$ & $-0.49+0.13 \mathrm{j}$ \\
\hline $2^{\text {nd }}$ & $0.92+073 \mathrm{j}$ & $0.28+0.11 \mathrm{j}$ & $-0.92-0.73 \mathrm{j}$ \\
\hline $3^{\text {rd }}$ & $-1.44+3.64 \mathrm{j}$ & $0.64+0.03 \mathrm{j}$ & $1.44-3.64 \mathrm{j}$ \\
\hline $4^{\text {th }}$ & $-0.97-2.23 \mathrm{j}$ & $-0.65+0.15 \mathrm{j}$ & $-0.97-2.23 \mathrm{j}$ \\
\hline
\end{tabular}
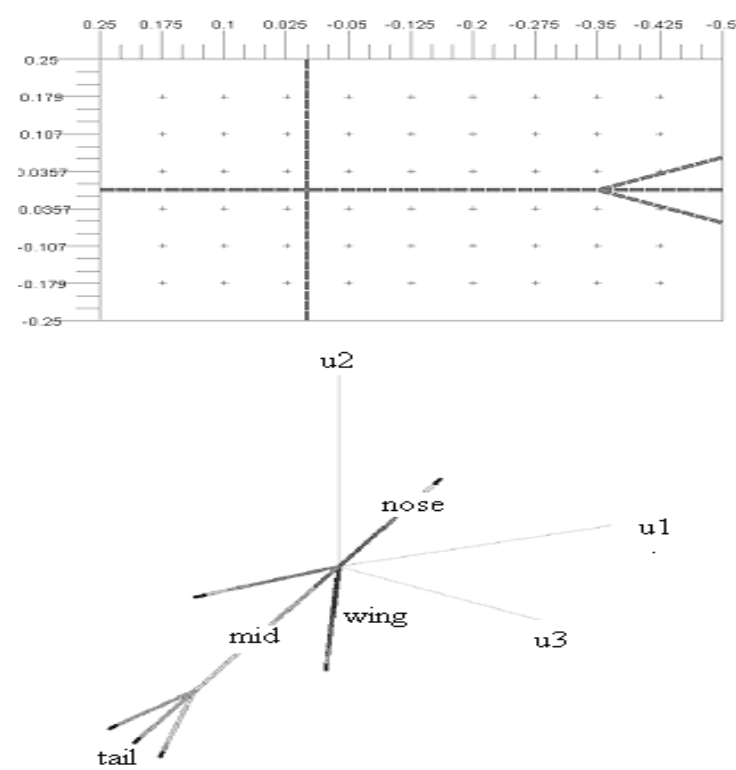

Figure 1: (Top inset) The geometries(in $\mathrm{cm}$ ) of the aircraft model. The aircraft coordinates oriented by $45^{\circ}$ about the $\mathrm{u}_{1}$-axis, with the wings having dihedral angles of $45^{\circ}$ each

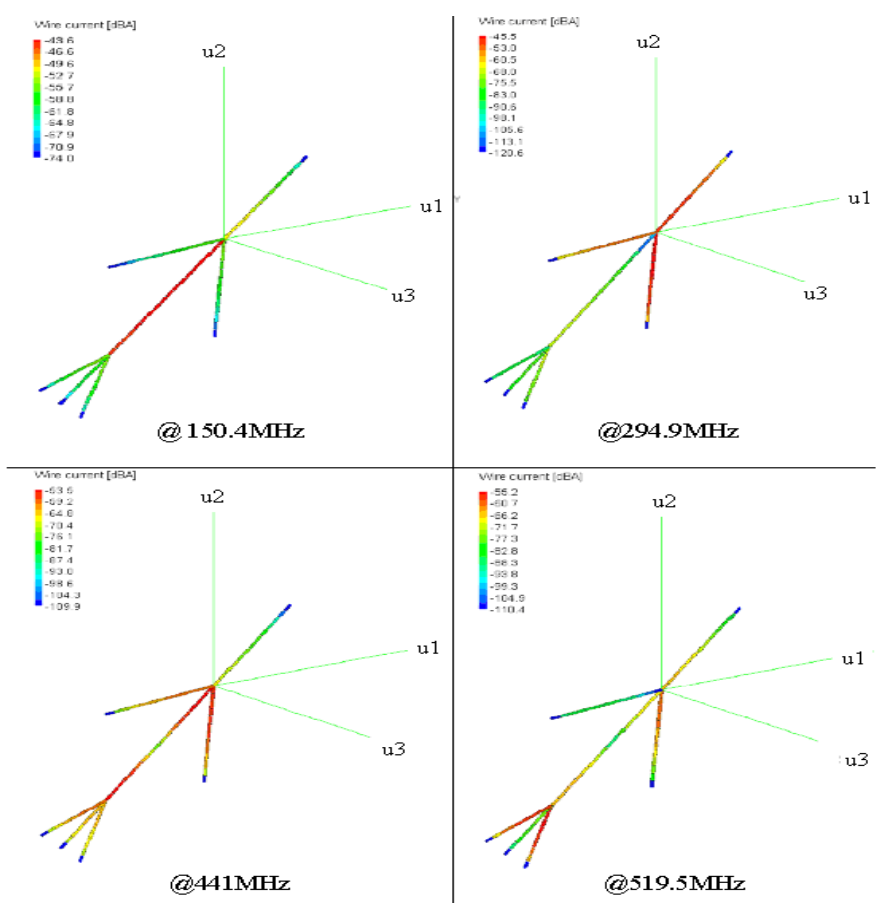

Figure2: The current distribution for different resonant frequencies. In general, the dominant modes are related to a part of the model structure

Table III: The Co-null CPS reflects the target composite.

\begin{tabular}{|c|c|c|c|c|c|c|c|}
\hline $\begin{array}{l}\text { Mode } \\
\text { order }\end{array}$ & CPS & $g_{1}$ & $g_{2}$ & $g_{3}$ & $\begin{array}{c}\text { Elongation } \\
\left|\left(\mathrm{g}_{\mathrm{cn} 1} \bullet \mathrm{g}_{\mathrm{cn} 2}\right)\right|\end{array}$ & $\begin{array}{c}\text { Symmetry } \\
1 / 2 \arcsin \\
\left(\mathrm{g}_{3}\right) \\
\end{array}$ & $\begin{array}{c}\text { Tilt } \\
1 / 2 \arctan \\
\left(\mathrm{g}_{2} / \mathrm{g}_{1}\right) \\
\end{array}$ \\
\hline \multirow{2}{*}{1} & $\mathrm{cn}_{1}$ & \begin{tabular}{|l|}
0 \\
\end{tabular} & -1 & 0 & \multirow{2}{*}{1} & \multirow{2}{*}{ 0 } & \multirow{2}{*}{45} \\
\hline & $\mathbf{c n}_{2}$ & 0 & -1 & 0 & & & \\
\hline \multirow{2}{*}{2} & $\mathbf{c n}_{1}$ & \begin{tabular}{|l|}
$\mathbf{0}$ \\
\end{tabular} & -0.26 & 0.97 & \multirow{2}{*}{0.87} & \multirow{2}{*}{ 0 } & \multirow{2}{*}{45} \\
\hline & $\mathrm{cn}_{2}$ & 0 & -0.26 & -0.97 & & & \\
\hline \multirow{2}{*}{3} & $\mathbf{c n}_{1}$ & 0 & -0.16 & 0.98 & \multirow{2}{*}{0.93} & \multirow{2}{*}{ 0 } & \multirow{2}{*}{45} \\
\hline & $\mathbf{c n}_{2}$ & \begin{tabular}{|l|l|} 
\\
\end{tabular} & $\mid-0.16$ & -0.98 & & & \\
\hline \multirow{2}{*}{4} & $\mathbf{c n}_{1}$ & \begin{tabular}{|l|}
$\mathbf{0}$ \\
\end{tabular} & -0.27 & \begin{tabular}{|l|}
0.96 \\
\end{tabular} & \multirow{2}{*}{0.88} & \multirow{2}{*}{ 0 } & \multirow{2}{*}{45} \\
\hline & $\mathbf{c n}_{2}$ & 0 & -0.27 & -0.96 & & & \\
\hline
\end{tabular}




\section{International Journal of Science and Research (IJSR) \\ ISSN (Online): 2319-7064}

Index Copernicus Value (2013): 6.14 | Impact Factor (2015): 6.391

\section{Conclusions}

The theory of SEM makes it feasible to extend the CPS concept from its original narrowband perspective into a broadband perspective. Extracted by MPOF, a single dominant mode can convey some part of the target composite or attributes, but not all. The residue terms of the mode have the capacity to reflect the polarization characteristics of the mode, and subsequently, the target composite or shape if all dominant modes are included. Specifically, the null states of the co-pol CPS can reflect the degrees of elongation, symmetry and tilt in the target composite. Henceforth, it is even possible based on the modal co-null set to discriminate between electrically similar targets, where resonance only methods face degradation. Finally, investigating the identification performance of this feature set in presence of noise and for multiple aspects can be a subject of further studies.

\section{Acknowledgement}

This work was supported and funded by The Public Authority of Education and Training, Research project No (TS-11-14).

\section{References}

[1] H. S. Lui and N. V. Z. Shuley, "Radar Target Identification Using a "Banded" E-pulse Technique," Antennas and Propagation, IEEE Transactions on, vol. 54, pp. 3874-3881, 2006.

[2] D. Lan, L. Hongwei, B. Zheng, and Z. Junying, "A two-distribution compounded statistical model for Radar HRRP target recognition," IEEE Transactions on Signal Processing, , vol. 54, pp. 2226-2238, 2006.

[3] W. Dangwei, M. Xinyi, and S. Yi, "Radar target identification using a likelihood ratio test and matching pursuit technique," Radar, Sonar and Navigation, IEE Proceedings -, vol. 153, pp. 509-515, 2006.

[4] K.-T. Kim, D.-K. Seo, and H.-T. Kim, "Radar target identification using one-dimensional scattering centres," Radar, Sonar and Navigation, IEE Proceedings -, vol. 148, pp. 285-296, 2001.

[5] D. A. Garren, A. C. Odom, M. K. Osborn, J. S. Goldstein, S. U. Pillai, and J. R. Guerci, "Full-polarization matched-illumination for target detection and identification," Aerospace and Electronic Systems, IEEE Transactions on, vol. 38, pp. 824-837, 2002.

[6] W. M. Steedly and R. L. Moses, "High resolution exponential modeling of fully polarized radar returns," Aerospace and Electronic Systems, IEEE Transactions on, vol. 27, pp. 459-469, 1991.

[7] P. B. Silverstein, O. S. Sands, and F. D. Garber, "Radar target classification and interpretation by means of structural descriptions of backscatter signals," Aerospace and Electronic Systems Magazine, IEEE, vol. 6, pp. 3-7, 1991.

[8] F. Aldhubaib, N. V. Shuley, and H. S. Lui, "Characteristic Polarization States in an Ultrawideband Context Based on the Singularity Expansion Method,"
IEEE Geoscience and Remote Sensing Letters, vol. 6, pp. 792-796, 2009.

[9] F. Aldhubaib, "Polarization Angles As A Radar Feature Set " International Journal of Enhanced Research in Science Technology \& Engineering (IJERSTE), vol. 5, April - 20162016.

[10]F. Aldhubaib and N. V. Shuley, "Radar Target Recognition Based on Modified Characteristic Polarization States," IEEE Transactions on Aerospace and Electronic Systems, vol. 46, pp. 1921-1933, 2010.

[11]F. Aldhubaib, H. S. Lui, N. V. Shuley, and A. Al-Zayed, "Aspect segmentation and feature selection of radar targets based on average probability of error," IET Microwaves, Antennas \& Propagation, vol. 4, pp. 1654-1664, 2010.

[12]F. F. H. Aldhubaib and N. V. Z. Shuley, "Characteristic Polarization States Estimation in an Ultrawideband Context: A Frequency Approach," IEEE Transactions on Geoscience and Remote Sensing, vol. 47, pp. 2808-2817, 2009.

[13]FA Sadjadi, CSL Chun, A Sullivan, and G. Gaunaurd, "The Huynen-Fork Polarization Parameters in the Classification of Dielectric Mine-like Objects," Sensing and Imaging: An International Journal, vol. 7, 2006.

[14] W.-M. Boerner, W.-L. Yan, A.-Q. Xi, and Y. Yamaguchi, "On the basic principles of radar polarimetry: the target characteristic polarization state theory of Kennaugh, Huynen's polarization fork concept, and its extension to the partially polarized case," Proceedings of the IEEE, vol. 79, pp. 1538-1550, 1991.

[15]H. Mott, Remote sensing with polarimetric radar New York, N.Y: Wiley-IEEE ; Chichester : John Wiley 2007.

[16] Fawwaz T. Ulaby and C. Elachi, Radar polarimetry for geoscience applications: Norwood, Mass. : Artech House, 1990.

[17] C. E. Baum, E. J. Rothwell, K.-M. Chen, and D. P. Nyquist, "The singularity expansion method and its application to target identification," Proceedings of the IEEE, vol. 79, pp. 1481-1492, 1991.

[18] C. E. Baum, "Signature-based target identification and pattern recognition," Antennas and Propagation Magazine, IEEE, vol. 36, pp. 44-51, 1994.

[19]E. s. a. systems, "Feko Suit 5," 9.3.24 ed. S.A (Pty) Ltd, 2003-2005.

\section{Author Profile}

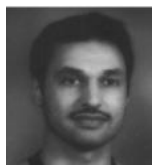

Faisal F. H. Aldhubaib received his B.E. degree in electrical and computer engineering and thenM.Sc.degreein radio and microwave engineering from the University of Leeds, UK, in 1995 and 1997, respectively. Then in 2010 , he received his $\mathrm{PhD}$ in Electromagnetic and Imaging research at the School of Information Technology and Electrical Engineering (ITEE) from the University of Queensland, Australia. Currently, he is an assistant professor working in the college of technological studies, PAAET, Kuwait. 